\title{
Au rendez-vous des cartographes
}

\author{
Bertrand R. Jordan
}

Les laboratoires de génétique humaine qui veulent figurer dans la compétition internationale s'adjoignent maintenant des collaborateurs spécialisés dans l'ingéniérie et la robotique : il n'y a plus guère de place pour le «bricolage » artisanal. Un formidable défi auquel la France ne semble pas s'être donné tous les moyens de répondre.
Nous avons parlé l'année dernière $\left(\mathrm{m} / \mathrm{s} n^{\circ} 7\right.$, vol. 4, p.448) de la première réunion sur la cartographie et la séquence du génome tenue à Cold Spring Harbor en avril 1988. Un an après, la deuxième édition de ce petit congrès est une bonne occasion de mesurer les progrès accomplis entretemps, au cours d'une période marquée par le début de la mise en place du projet de séquençage du génome humain aux Etats-Unis - et par la poursuite (on pourrait même dire l'enlisement) des discussions préparatoires au lancement d'un tel programme en Europe et au Japon. Plus de deux cents chercheurs dans ce domaine se retrouvaient donc, autant pour faire le point sur les mérites relatifs des différentes façons de déterminer la carte physique et la séquience de très grands segments d'ADN que pour confronter les résultats obtenus avec ces méthodes.

Le scoop de l'an dernier avait été l'annonce par Bob Moysis (Los Alamos, New Mexico, USA) du clonage de la séquence constituant les télomères humains. Cette année, toute une session était consacrée aux développements de ce travail. Un point fort: le clonage de grands segments comprenant le télomère mais aussi des séquences uniques subtélomériques par la tactique du «demiYAC* $^{*}$ ». En utilisant pour le clonage dans la levure d'ADN humain un seul bras du vecteur YAC, on sélectionne des segments contenant des séquences télomériques humaines,

\section{TIRÉS A PART}

B.R. Jordan.

\footnotetext{
* YAC : yeast artificial chromosome.
}

qui peuvent fonctionner aussi comme télomères dans la levure [ 1 , 2]. Parmi les séquences adjacentes à la séquence télomérique proprement dite (qui est commune à tous les chromosomes), on peut trouver des séquences uniques qui fournissent alors une sonde subtélomérique spécifique d'un chromosome donné. De telles séquences sont précieuses pour la cartographie physique en général mais aussi, et tout particulièrement, pour le chromosome 4 où le gène responsable de la chorée de Huntington (toujours recherché !) est tout près du télomère du bras court. Les télomères eux-mêmes, mieux connus maintenant, montrent de curieuses variations ; ils semblent se raccourcir au cours du développement, et rallonger dans certaines cellules tumorales. Ce phénomène, peut-être important pour la durée de vie des cellules, motive aussi des études sur l'enzyme responsable de ces modifications : cette enzyme vient d'être isolée à partir de cellules humaines HeLa.

Le reste du colloque a été en grande partie centré autour de progrès technologiques et de leur exploitation. Le clonage d'ADN humain sous forme de chromosomes artificiels dans la levure YAC [3] a tenu encore une fois la vedette. A juste titre puisqu'il s'agit pour le moment du seul système capable de cloner des segments de 200, 300 voire 700 kilobases, mais aussi parce que - contrairement à l'an dernier - il est maintenant en place et en cours d'exploitation dans plusieurs laboratoires. Les groupes les plus avancés, en particulier ceux de Maynard Olson et David Schlessinger à Saint Louis, Mi, USA 
- sans oublier Hans Lehrach à l'ICRF* Londres, GB - ont mis au point des techniques de manipulation, de stockage et de criblage efficaces, en particulier (Eric Green, Saint-Louis, Mi, USA) une très jolie technique de criblage par PCR (polymerase chain reaction) sur le mélange d'ADN de près de deux mille clones différents qui permet de trouver avec un minimum d'efforts le clone correspondant à une sonde donnée, à condition d'avoir fait un peu de séquence sur cette sonde pour pouvoir choisir les amorces **. Les librairies construites à Saint Louis (USA) sont en cours d'exploitation. L'une d'elles, qui couvre uniquement le tiers du bras long du chromosome $\mathrm{X}$ (Xq24-Xq28), semble à peu près complète et fournit un flot de clones qui devraient bientôt couvrir l'ensemble de cette région, et donc rendre beaucoup plus accessible l'approche par la « génétique inverse » d'un certain nombre de maladies génétiques localisées dans cette zone.

Une autre technologie, celle de l'hybridation in situ à l'aide de sondes «froides» (fluorescentes at non radioactives) a fait de très grands progrès. Le principe n'est pas nouveau : remplacer la détection radioactive d'une sonde fixée sur un chromosome par celle d'une molécule fluorescente qui se fixe sur la sonde préalablement modifiée. Mais cette méthode, qui a longtemps souffert d'un manque de sensibilité, permet maintenant d'avoir de bons résultats avec une sonde simple copie longue de 5 kilobases seulement - et surtout l'utilisation directe comme sonde de cosmides ou même de YACs (en présence d'ADN total compétiteur pour «éteindre » le signal parasite dû aux séquences répétées) est maintenant très au point. Les avantages sont énormes : localisation très fine, puisqu'on détecte un fluorochrome collé sur la sonde, elle-même collée sur le chromosome, et non un gros grain d'argent produit dans l'émulsion photographique à une distance de quelques micromètres. De plus, l'efficacité actuelle de ces techniques est proche de $100 \%$, c'est-à-dire que dans chaque métaphase, les deux chromatides du chromosome concerné sont marquées: l'exploitation statistique des résultats n'est quasiment plus nécessaire. On peut utiliser simulta- nément deux ou plusieurs sondes avec - comble du raffinement - des couleurs différentes; on peut « peindre » un chromosome donné, et lui seul, par un mélange de sondes spécifiques. On peut (en microscopie confocale $^{* * *}$ ) quantifier et travailler l'image... Bref, une gamme très nouvelle de possibilités dont Peter-Pearson (Leiden, Pays Bas) et David Ward (Yale, Ct, USA) ont démontré l'intérêt: localisation in situ de 50 cosmides en une semaine, étude détaillée et rapide d'aberrations chromosomiques... Le «clou » de la session a été la présentation par Barbara Trask (Livermore, Ca, USA) d'hybridations in situ sur des noyaux en interphase dans lesquels l'ADN est relativement peu compacté : deux sondes distantes de $100 \mathrm{~kb}$ seulement donnent deux points distincts. On imagine les applications possibles en cartographie précise à l'intérieur d'une bande chromosomique (une bande équivaut en moyenne à $5000 \mathrm{~kb}$ ) surtout dans un système à plusieurs couleurs.

Le jeu des contigs (voir $\mathrm{m} / \mathrm{s} n^{\circ} 7$, vol. 4, p. 448) consiste à couvrir une région, un chromosome ou même un génome entier par un nombre approprié de cosmides dont on détermine les recouvrements par des variantes de plus en plus automatisées de la méthode des fingerprints de Coulson. $\mathrm{Ce}$ jeu continue de plus belle : le plus bel exemple est fourni justement par le groupe d'Alan Coulson, qui a déjà analysé 17500 cosmides pour couvrir le génome du nématode Cœnorhabditis elegans (100 Mb soit presque la taille du chromosome X humain). Les cosmides assemblés en contigs couvrent maintenant $90 \%$ du génome et il n'y a plus «que » 228 trous grâce à l'aide d'une librairie auxiliaire dans les YACs [4] qui a permis d'en combler près de 500 . Ce type de travail est activement poursuivi par le groupe d'Antony Carrano (Livermore, $\mathrm{Ca}$, USA) sur le chromosome 19, de Bob Moysis (Los Alamos, New Mexico, USA) sur le

\footnotetext{
* ICRF : imperial cancer research fund.

* Voir $m / s, n^{\circ} 8$, vol. 4, p. 515

** Confocale : technique évoluée de microscopie optique permettant le recueil et le traitement d'information beaucoup plus précises qu'en microscopie classique.
}

chromosome 16, de Glen Evans (Salk Institute, San Diego, Ca, USA) sur le bras long du chromosome ll, de Steve Warren (Atlanta, Ca, USA) sur la bande q28 du chromosome X... Dans chaque cas, ce travail énorme est en partie automatisé tant dans l'exécution des manipulations préalables (préparations d'ADN, coupure, chargement des gels), domaine où le robot Beckman ${ }^{\circledR}$ plus ou moins modifié se taille un beau succès, que pour la séparation des fragments (utilisation «détournée » des séquenceurs automatiques) ou pour l'interprétation des données et la détermination des recouvrements entre cosmides. On est très loin de l'artisanat et, dans chaque cas, ces mises au point sont le fruit de collaborations avec des départements d'ingénierie et/ou des industriels. Les contigs avouent parfois leurs limites: zones inclonables ou trop riches en séquences répétées - mais de plus en plus les YAC sont utilisés pour prendre la relève et jeter des ponts entre les îlots déjà construits. Il ne faudra pas attendre longtemps, sans doute, pour voir apparaître la première série complète de cosmides couvrant une bande chromosomique entière, qui pourrait bien être la bande Xq28. Après tout cela ne représente que le double ou le triple du génome d'Escherichia coli pour lequel le travail a été fait à la main par trois chercheurs japonais il y a déjà deux ans [5] !

Pour rester encore dans le domaine des technologies, différentes «astuces» pour détecter une mutation dans un gène sans savoir a priori où elle se trouve ont été présentées. Ces méthodes font en général appel à la PCR pour, tout d'abord, amplifier la région à étudier à partir d'un peu d'ADN du malade. La présence d'une mutation dans cette région peut alors être révélée par une électrophorèse dans un gel à gradient de dénaturation (migration extrêmement sensible à la séquence), par l'incorporation d'analogues comıme des nucléotides biotinylés ( $T$, par exemple, provoquant une importante différence de mobilité si, à cause de la mutation, un $\mathrm{T}$ supplémentaire est présent), ou par fixation et tentative de ligation de deux oligonucléotides adjacents (la ligation échouera s'il existe une mutation au 


\section{RÉFÉRENCES}

1. Cross SH, Allshire RC, McKay SJ, McGill NI, Cooke HJ. Cloning of human telomeres by complementation in yeast. Nature 1989; $338: 771-4$.

2. Brown WRA. Molecular cloning of human telomeres in yeast. Nature $1989 ; 338$ : 774-6.

3. Burke DT, Carle GF, Oslon MV. Cloning of large segments of exogenous DNA into yeast by means of artificial chromosome vectors. Science 1987 ; 236 : 806-12.

4. Coulson A, Waterson R, Kiff J, Sulston J, Kohara Y. Genome linking with yeast artificial chromosomes. Nature 1988 ; 335 : 184-6.

5. Kohara Y, Akiyama K, Isono K. The physical map of the whole $E$. coli chromosome: application of a new strategy for rapid analysis and sorting of a large genomic library. Cell 1987 ; 50 : 495-508.

\section{Summary}

When cartographers meet...

Developments in genome mapping (mostly) and sequencing (a little) were reviewed in a recent meeting at Cold Spring Harbor (April 89). Salient scientific features are the cloning of human chromosome specific subtelomeric sequences, continued and intensive use of cosmid and YAC libraries with automated assembly of overlapping cosmids into «contigs», quick and sensitive localization of DNA probes by in situ hybridization of whole cosmids or YACs labelled with fluorescent probes, and an array of efficient methods to detect single base mutations without prior knowledge of where the mutation lies exactly. This accelerated pace of technological change takes place mostly in the US and raises serious questions for European countries like France in which such developments have been slow to materialize. niveau de la jonction entre ces deux séquences). Ces techniques, de même que celle qui détecte le non-appariement d'une seule base par action du tétroxyde d'osmium et de l'hydrazine, allient rapidité et possibilité d'automatisation; l'arrière-pensée évidente de leurs promoteurs est de les introduire comme technique de routine en milieu hospitalier. Quant à la séquence de l'ADN, qui occupe la moitié du titre du colloque (genome mapping and sequencing), elle n'a pris qu'une place limitée (une session) consacrée à passer en revue les performances actuelles de ces systèmes, avec une mention particulière pour la machine de l'EMBL (european molecular biology laboratory) qui a été utilisée pour séquencer l'ensemble du locus HPRT, soit 60 kilobases (Wilhelm Ansorge, EMBL, Heidelberg, RFA, et David Nelson, Baylor College, Houston, Tx, USA). La « GENOMYX Corporation » (Thomas Brennan, San Francisco, $\mathrm{Ca}, \mathrm{USA}$ ) nous a fait rêver avec un système de séquence par électrophorèse en capillaire d'ADN marqué par quatre isotopes stables du soufre, avec détection par spectrométrie de masse. C'est une méthode apparemment fiable, rapide et surtout hyperrésolutive, potentiellement capable de «lire» la séquence jusqu'à 1000 ou 2000 bases sur le même fragment - mais elle en est à une phase exploratoire.

On a tout de même un peu parlé des maladies génétiques à ce colloque avec, en particulier, les présentations de Francis Collins (Ann Arbor, USA) sur la mucoviscidose et de David Cox (San Francisco, Ca, USA) sur la chorée de Huntington. En bref, le gène impliqué n'est encore isolé ni dans un cas ni dans l'autre, mais le filet se resserre : pour la mucoviscidose, la région à explorer est maintenant réduite à environ 300 kilobases et est déjà largement garnie en sondes ; pour la chorée de Huntington, et à moins d'anomalies de recombinaison toujours possibles (d'autant plus que l'on est tout près du télomère), le gène doit être dans un segment de 200 kilobases allant jusqu'au télomère du bras court du chromosome 4 - segment dont le clonage en tant que YAC a été annoncé par Hans Lehrach (ICRF, Londres, GB). Reste évidemment à identifier le gène et à prouver que c'est bien lui le responsable, chose déjà préparée pour la mucoviscidose avec les études de canaux ioniques, mais plus difficile à concevoir pour la chorée de Huntington dans la mesure où l'on ne sait pas dans ce cas comment se manifeste le défaut au niveau cellulaire.

En conclusion et au-delà de tel ou tel aspect des résultats ou des techniques présentés à ce colloque, une impression domine, c'est celle d'assister à une double révolution technologique. Les méthodologies nouvelles amplification par PCR, analyse en champs pulsés, librairies YAC dans la levure, pour ne citer qu'elles occupent à l'évidence une place de plus en plus importante et cela d'autant plus qu'elles sont fructueusement combinées entre elles. Mais de plus la façon dont sont mises en œuvre ces méthodes change: l'échelle, la rapidité, la technicité des manipulations de laboratoire sont en train de subir un saut qualitatif. Sous l'effet, en particulier, du programme Génome Humain (toujours dans les limbes en Europe, faut-il le rappeler) la robotique fait une entrée en force dans le laboratoire de biologie moléculaire, par exemple pour la préparation, l'analyse et l'assemblage de cosmides en contigs, ou pour la manipulation de librairies dans la levure. Des incitations multiples à la collaboration entre de tels laboratoires et des départements ou des entreprises de technologie, dans un milieu déjà plus favorable que le nôtre à de tels croisements, ont abouti à un accroissement d'un ordre de grandeur ou plus de la productivité pour un certain nombre d'opérations de génie génétique.

Cela pose de sérieux problèmes aux Européens, et aux Français en particulier dont la morosité à ce colloque était nettement perceptible. Cette débauche de technologie et ce saut qualitatif dans la productivité de recherche rendent en effet caducs des travaux comparables faits à la main, même s'ils sont ciblés sur un gène ou une région chromosomique particulière. De plus le problème n'est pas limité au génome humain dans la mesure où ces méthodes commencent déjà à être appliquées à l'étude d'organismes dont l'intérêt est $a$ priori plus «académique » (la droso- 
phile par exemple) ou au contraire plus appliqué (le végétal). Il ne faudrait pas non plus se laisser bercer par l'illusion que nous pourrions faire l'impasse sur ce «travail de structure stupide » pour, une fois les chromosomes cartographiés, les cosmides alignés et - à la limite - la séquence du génome faite, utiliser tranquillement ces clones et ces résultats pour faire de la «vraie » recherche (plus proche de la fonction). Il était très clair, et cela a été dit explicitement au moins dans les conversations privées, que ceux qui n'auront pas sérieusement contribué à l'obtention de ces matériaux et de ces connaissances n'y auront qu'un accès limite, et tardif, quand les développements les plus intéressants au niveau fondamental ou appliqué auront déjà été exploités.

Il paraît évident qu'il faut dans ces conditions réagir très rapidement tant au niveau français qu'européen définir des buts réalistes (inutile d'essayer de courir après les Etats-Unis dans tous les domaines à la fois) et appliquer une politique à la fois réellement incitative au niveau des crédits et sérieusement directive pour éviter la dispersion des efforts. Il faut aussi faire passer dans les faits une transdisciplinarité biologique-physique dans les domaines de la robotique et de l'instrumentation, dont on parle beaucoup mais qui n'est guère soutenue dans le concret. Et même si le problème n'est pas seulement financier, les moyens assez limités dégagés jusqu'à maintenant dans notre pays et, au niveau européen, l'incapacité à ce jour à décider le démarrage effectif d'un projet de «médecine prédictive» pourtant bien modeste ( 15 millions d'ECU seulement, soit environ 17 millions de dollars sur trois ans face à 100 millions de dollars aux EtatsUnis pour un an) n'incitent malheureusement pas à l'optimisme...

Bertrand Jordan

Directeur de recherche au Cnrs, responsable du groupe "génétique moléculaire humaine". CIML, Inserm/Cnrs, case 906, 13288 Marseille Cedex 9, France. 